\title{
Electron spin resonance spectroscopic demonstration of the generation of reactive oxygen species by diseased human synovial tissue following ex vivo hypoxia-reoxygenation
}

\author{
D Singh, N B Nazhat, K Fairburn, T Sahinoglu, D R Blake, P Jones
}

\begin{abstract}
Objective-To apply an electron spin resonance (ESR) spectroscopic technique as a means of determining the oxidising capacity of reactive oxygen species produced during hypoxia and reoxygenation of diseased human synovial tissue.

Methods-Twenty four specimens of fresh synovial tissue were obtained from patients undergoing primary total knee joint replacement and graded according to the degree of inflammation present. Tissue samples were subjected to an ex vivo hypoxia-reoxygenation cycle in the presence of the nitroso based spin trap, 3,5-dibromo-4-nitrosobenzene sulphonate. The degree of oxidation of the spin trap to a stable free radical was determined and followed with time. Control samples were subjected to hypoxia only.
\end{abstract}

Results-The results indicate that the oxidising capacity of reactive oxygen species produced by human synovial tissue varies with the degree of inflammation present. Only the more inflamed specimens, from both rheumatoid arthritis and osteoarthritis patients, demonstrated increased production of reactive oxygen species when subjected to a hypoxia-reoxygenation cycle. This change was reduced by both competitive and non-competitive inhibitors of the endothelial based enzyme xanthine oxidase. The relative concentration of reactive oxygen species generated by the synovial tissue samples correlated with the mean capillary density of the specimens.

Conclusion-This study supports the hypothesis of movement induced hypoxicreperfusion injury of the chronically inflamed joint by demonstrating the generation of reactive oxygen species within inflamed human synovium following an ex vivo hypoxia-reoxygenation cycle. Evidence is presented that the microvascular endothelial based enzyme xanthine oxidase is the predominant source of ESR detectable oxidising species in inflamed synovial specimens exposed to hypoxia-reoxygenation.

(Ann Rheum Dis 1995; 54: 94-99)
We have previously suggested that the persistence of joint inflammation may in part result from exercise induced hypoxicreperfusion cycles, with the consequent generation of reactive oxidising species. ${ }^{1-3}$ Many cells present in the inflamed synovium, including macrophages, neutrophils, and endothelial cells, have the capacity to produce reactive oxygen species. When such cells are isolated and stimulated they may be demonstrated to promote oxidative damage to biomolecules. $^{3}$ Oxidative modification to proteins, including alpha-1-antitrypsin ${ }^{4}$ and immunoglobulin, ${ }^{5}$ polysaccharides (such as hyaluronic acid) ${ }^{6}$ and lipid 7 can be detected in synovial fluid obtained from the inflamed joint, with the degree of modification increasing following exercise. Both normal and rheumatoid synovia contain xanthine oxidase/ dehydrogenase activity, localised to the capillary endothelium. ${ }^{89}$ The oxidase form of this enzyme is capable of generating reactive oxygen species following hypoxic-reperfusion cycles. ${ }^{10}$

Attempts to identify the production of reactive oxygen species within the inflamed joint have so far relied upon indirect evidence provided by assays of oxidative damage. We have now applied an electron spin resonance (ESR) spectroscopic technique as a means of assessing the ability of diseased human synovial tissue to generate oxidising species under conditions of hypoxia and hypoxiareoxygenation. ESR spectroscopy is a method of detecting free radicals ${ }^{11}{ }^{12}$ which exploits the magnetic properties of the unpaired electron(s) in free radical species. In this study the nitroso based compound 3,5-dibromo-4-nitrosobenzene sulphonate (DBNBS), previously shown to be non-toxic, ${ }^{13}$ has been used.

DBNBS may be used as a conventional spin trap because it forms more stable adducts with short lived radicals, such as the methyl radical $\left({ }^{\circ} \mathrm{CH}_{3}\right)$, which are subsequently detectable by ESR spectroscopy. However, DBNBS may also be used to detect oxidising species which are capable of forming the radical cation $\mathrm{DBNBS}^{+\cdot}$ by one electron oxidation. The three line ESR spectrum of this species has been characterised in the horseradish peroxidase catalysed oxidation of DBNBS by hydrogen peroxide, ${ }^{14}$ a one electron peroxidatic oxidation similar to that of 2,2'-azinobis(3ethyl benzthiazoline-6-sulphonic acid), as described by Wolfenden et al. ${ }^{15}$ Flitter and 
Mason $^{16}$ have similarly assigned the three line spectrum generated in such experiments to an oxidation product of DBNBS. In accordance with this assignment, the ESR signal is abolished by reduction with ascorbic acid. ${ }^{17}$

In the present study, immunohistochemical analysis was also performed to examine potential relationships between the clinical scoring system used (based on thickness and colour of the synovium), histological features, and the oxidising capacity of reactive oxygen species produced by the synovial tissue specimens following hypoxia-reoxygenation. In view of the potential critical importance of the enzyme xanthine oxidase/dehydrogenase to the process of hypoxic-reperfusion and its localisation to the synovial endothelium, ${ }^{8}$ we chose to stain our sections in order to identify the vascular endothelium and allow an assessment of vessel density.

In addition, using this method we have assessed the capacity of oxypurinol (a competitive inhibitor of xanthine oxidase $)^{18}$ and amflutizole (a non-competitive inhibitor), ${ }^{19}$ to limit the generation of synovial tissue derived oxidising species following ex vivo hypoxia-reoxygenation.

\section{Patients and methods}

This study was approved by the London Hospital Medical College Ethical Committee.

Patients from whom samples were obtained were all undergoing primary total knee joint replacements at the Royal London Hospital. Of the 24 patients studied (age range 48-82), 15 had primary osteoarthritis and nine had rheumatoid arthritis, as diagnosed by the referring physician. Samples of tissue were obtained from the suprapatellar and fat pad regions of the knee joint at surgery. The tourniquet used for the prevention of operative blood loss had been applied for an average of 11 (SD 6) minutes before sample collection. Time from sampling to commencement of the experimental protocol was approximately 30 minutes. The tissue samples were graded clinically as being inflamed or less inflamed: a numerical score for colour (red $=2$; pink $=1$; white $=0$ ) and thickness (proliferative $=2$; normal thickness $=1$; atrophic $=0$ ) defined the extent of the inflammatory reaction clinically; samples with a combined score greater than 2 were labelled 'inflamed' and the others were labelled 'less inflamed'.

Fresh tissue samples were immediately dissected to separate the synovium from adjacent capsule or fatty tissue. The synovial tissue was then cut into small pieces and two samples of $0.3 \mathrm{~g}$ of tissue (wet weight) were prepared for each experiment. Each tissue sample was immersed in $0.6 \mathrm{ml}$ phosphate buffered saline (pH 7.4) and $0.4 \mathrm{ml}$ of freshly prepared DBNBS $(100 \mathrm{mmol} / \mathrm{l}$ to give a final concentration of $40 \mathrm{mmol} / \mathrm{l})$. The test tube containing the specimen in its surrounding medium was placed in a water bath kept at $37^{\circ} \mathrm{C}$.

Paired synovial tissue samples from each patient were exposed to 60 minutes of hypoxia by bubbling a gaseous mixture of $95 \%$ nitrogen $/ 5 \%$ carbon dioxide through a glass pipette into the surrounding bathing medium at a rate of 10-15 bubbles per minute. For the control sample the hypoxic challenge was continued for a further two hours, whilst after 60 minutes hypoxia the second sample was reoxygenated by changing the gaseous mixture of $95 \%$ air $/ 5 \%$ carbon dioxide. The gaseous concentrations were chosen to reflect the tissue concentration of oxygen and carbon dioxide in vivo.

Aliquots $(0.02 \mathrm{ml})$ of the bathing medium surrounding each tissue sample were removed at regular (10-15 minute) intervals and placed into capillary quartz sample tubes (JEOL ESLCO1) for ESR analysis. Each aliquot completely filled the capillary tube, which was immediately sealed and then positioned in the cylindrical cavity of a JEOL JES-RE1X $\mathrm{X}$-Band Spectrometer and the microwave unit adjusted. An internal standard (JEOL $\mathrm{Mn}^{2+}$ marker) was used to ensure reproducibility. The spectrometer parameters, apart from receiver gain, were kept constant in all our experiments: modulation amplitude $0.32 \mathrm{mT}$, microwave power $10 \mathrm{~mW}$, microwave frequency $9.44 \mathrm{GHz}$, time constant $0.01 \mathrm{~s}$, sweep width $10 \mathrm{mT}$ and sweep time $5 \mathrm{~s}$. In order to increase the signal to noise ratio, the signal was accumulated over $50-60$ scans using a computer program (supplied by Dr John G Smith of the Department of Chemistry at the University of Newcastle-upon-Tyne). The $\mathrm{g}$ value of the three line spectrum was measured using the signal from the standard $\mathrm{Mn}^{2+}$ marker of the JEOL instrument. ${ }^{14}$

Although, in principle, double integration of absorption first derivative ESR spectra yields an absolute method for determination of radical concentrations, the errors involved under other than ideal conditions (i.e. most practical conditions, particularly with biological samples) are considerable. An analytical calibration curve using first derivative peak height is a suitable alternative approach and we have, with the use of 2,2,6,6-tetramethyl1-piperidinyloxy (a stable free radical) shown this to be satisfactory in other work. In the present study, therefore, we have used peak heights as a measure of the relative concentrations of reactive oxygen species present. The height of the middle line of the spectrum was measured using the computer program. The signal height was normalised to arbitrary units to allow for differences in the receiver gain of the spectrometer in separate experiments.

Assessment of the effect of oxypurinol and amflutizole in this system was carried out on synovial tissue samples subjected to 30 minutes of hypoxia followed by 30 minutes of reoxygenation. Oxypurinol or amflutizole (both at a final concentration of $1 \mu \mathrm{mol} / \mathrm{l}$ ) was included in the incubation medium at the time of addition of DBNBS. Signal measurements were made 24 hours after termination of hypoxia-reoxygenation.

Suitable synovial tissue samples were available for histological analysis from six 
patients and were collected at the time of dissection of the synovial tissue before starting the ESR spectroscopic experiments. Four of the specimens were from patients with rheumatoid arthritis (two classified as inflamed and two classified as less inflamed) and two specimens were from patients with osteoarthritis (both less inflamed; complete data from an inflamed specimen were not available because of processing difficulties). The histological sections were stained immunohistochemically using a rabbit polyclonal antihuman von Willebrand Factor antibody (Dako) and Vectastain Elite ABC peroxidase enhancement kit (Vector Laboratories).

Images for morphometric analysis were obtained by outlining the morphological features in pencil on A4 paper using an Olympus BHT light microscope equipped with a drawing arm attachment at $\times 20$ objective lens magnification. Several diagrams of representative areas of each slide were drawn and each diagram was digitised using a TDS ${ }^{\circledR}$ LC A3 digitiser with a four button cursor. The data were collected and processed on an Opus PC V personal computer; the computer program has been written and described by Stevens et al. ${ }^{20}$

The morphological parameters assessed in order to investigate capillary spatial characteristics were capillary numerical density, calculated as the number of capillaries per unit area of synovium (the program only recognised vessels of less than $15 \mu \mathrm{m}$ as a capillary) and capillary depth, defined as the shortest distance between the synovial surface and the nearest point on the luminal aspect of the capillary endothelium. At the same time, the cell density of the specimen was assessed by giving each slide a score of,+++ , or +++ upon observation of the stained section using a $\times 4$ lens objective. As the cell density varied in different areas of the field of view at greater magnification, we did not draw individual cells for subsequent digitisation of the drawing.

\section{Results}

Of the 15 specimens from patients with primary osteoarthritis, 12 had a score equal to or less than 2 and were graded as less inflamed. The remaining three samples were graded as inflamed on our clinical score. Of the nine specimens from patients with rheumatoid arthritis, three were inflamed and six were less inflamed.

Figure 1 shows a representative record of a three line spectrum resulting from oxidation of the spin trap DBNBS and obtained with a specimen of inflamed rheumatoid synovial tissue at 90 minutes incubation. The spectrum exhibits a major three line signal from the oxidised DBNBS radical $(\mathrm{g}=2.0065$, $A_{N}=1.33 \mathrm{mT}$ ) superimposed on a weak background signal of unknown origin. The method of height measurement of the middle peak of the signal is also shown.

Figure 2A shows the change with time in the normalised signal height measurement produced with a specimen of less inflamed rheumatoid synovium (clinical score $=1$ ). With the control sample the degree of oxidation of the DBNBS, corresponding to the relative concentration of oxidising species produced by the synovium, gradually increased with time. Reoxygenation of the second sample from the same patient after 60 minutes made no appreciable difference to the rate of oxidation of the spin trap. Experiments on different pieces of synovium from the same patient gave reproducible results.

Figure 2B shows the results obtained with a specimen of inflamed rheumatoid synovial tissue (clinical score $=4$ ). The different scale should be noted for the normalised signal height measurements of figure $2 \mathrm{~B}$, reflecting the greater oxidation of the spin trap observed with the sample of inflamed rheumatoid synovial tissue in comparison with less inflamed rheumatoid synovium (figure 2A). Furthermore, in marked contrast to the results obtained with less inflamed synovium,

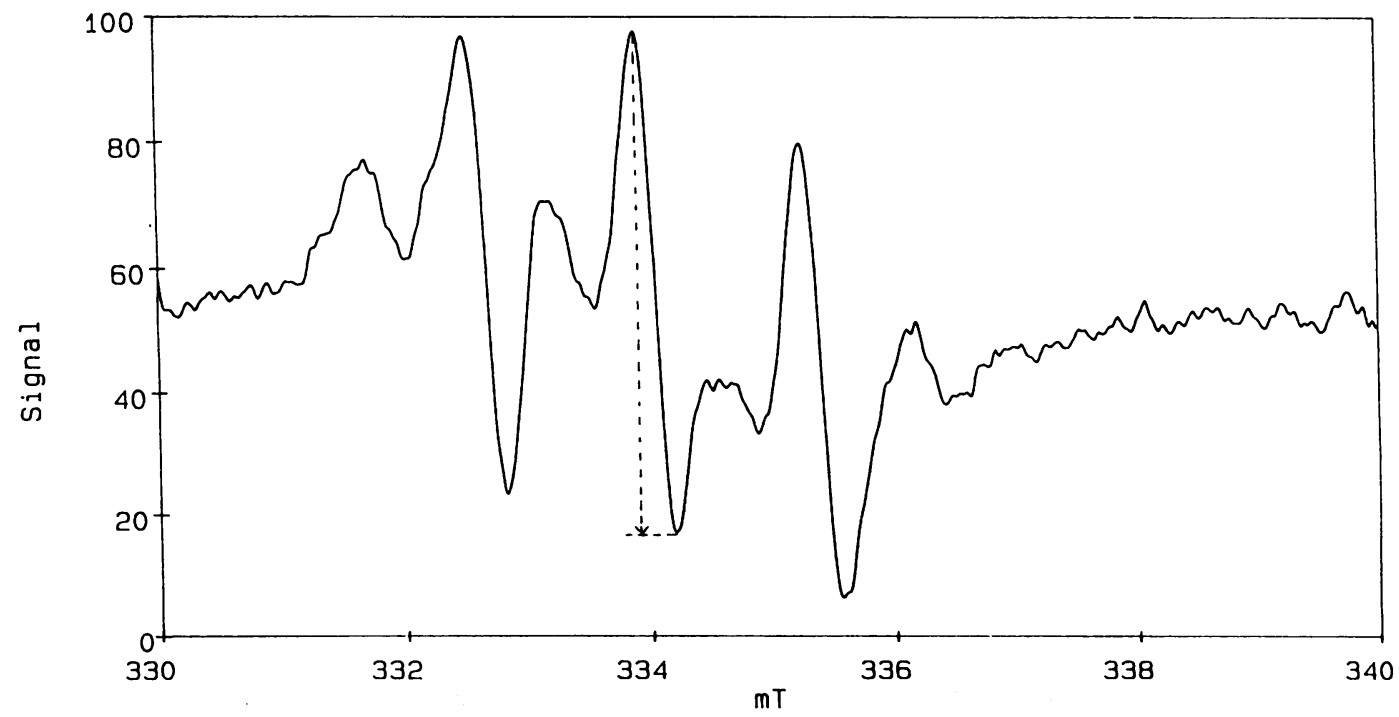

Figure 1 Representative ESR spectrum obtained with oxidised DBNBS. The spectrum exhibits a major three line signal from the oxidised DBNBS radical $\left(g=2.0065, A_{N}=1.33 \mathrm{mT}\right)$ superimposed on a weak background signal of unknown origin. The height of the middle line, as indicated, is measured by arbitrary units and is proportional to the concentration of the oxidised DBNBS. 

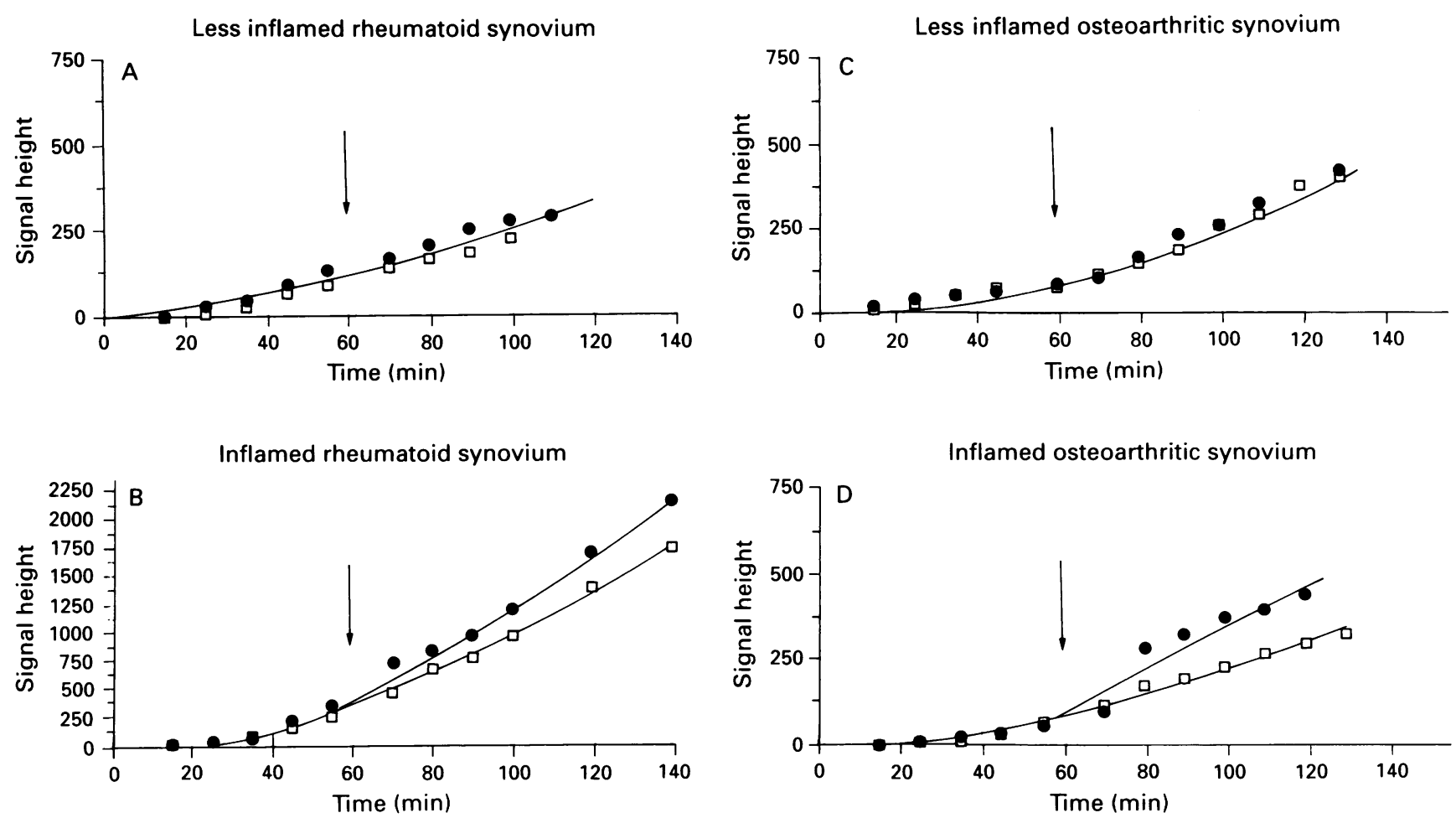

Figure 2 Signal height (arbitrary units) against time in four experiments using samples of synovium from four patients with the diagnosis and scoring of the degree of inflammation as indicated. Note the different scale in B. $\square=$ Hypoxia throughout; $\bullet=60$ minutes hypoxia followed by reoxygenation (arrows) of the tissue samples.

reoxygenation of inflamed rheumatoid synovium induced an increase in the rate of oxidation of the spin trap, in this instance resulting in a $28 \%$ increase in signal height at 120 minutes compared with the control experiment in which the paired sample was exposed to continuous hypoxia. Figures 2C and $2 \mathrm{D}$ show similar results with specimens of osteoarthritic synovium at both extremes of our clinical scoring of inflammation.

The degree of oxidation of the DBNBS was similar for less inflamed synovium from both rheumatoid $(n=6)$ and osteoarthritic patients $(n=12)$. In none of these samples was a change in the rate of oxidation of the DBNBS observed after reoxygenation compared with the paired control sample exposed to continuous hypoxia. However, for each inflamed sample obtained from both rheumatoid arthritis and osteoarthritis patients (combined total $\mathrm{n}=6$ ), an increase in the capacity of the synovium to oxidise the spin trap DBNBS was observed compared with the corresponding paired control sample exposed to continuous hypoxia. A control experiment of hypoxia-reoxygenation with DBNBS in

Table 1 Normalised signal height at 120 minutes for samples undergoing hypoxiareoxygenation cycle compared with control paired samples exposed to hypoxia alone

\begin{tabular}{llll}
\hline & \multicolumn{2}{l}{ Signal height (arbitrary units) } & p† \\
\cline { 2 - 3 } & Hypoxia only & Hypoxia-reoxygenation & \\
\hline $\begin{array}{l}\text { Less inflamed OA synovium } \\
(\mathrm{n}=12)\end{array}$ & $268(175-358)$ & $271(189-386)$ & 0.060 \\
$\begin{array}{c}\text { Less inflamed RA synovium } \\
(\mathrm{n}=6)\end{array}$ & $245(181-369)$ & $319(232-345)$ & 0.092 \\
$\begin{array}{c}\text { Inflamed RA and OA synovium } \\
(\mathrm{n}=6)\end{array}$ & $535(303-875)$ & $730(446-1155)$ & 0.009 \\
\hline
\end{tabular}

Values are medians and corresponding interquartile ranges.

†Paired sample $t$ test (within-sample differences had an approximately normal distribution). phosphate buffered saline without synovial tissue yielded no evidence for oxidation of the spin trap in the absence of tissue. Further control experiments performed using muscle tissue and adipose tissue from the same patients demonstrated no change in the rate of oxidation of the spin trap by these samples after hypoxia-reoxygenation.

Table 1 collates the normalised signal heights obtained from all 24 samples at 120 minutes from the start of the each experiment. Comparing the normalised signal heights for the less inflamed rheumatoid and osteoarthritic synovia undergoing hypoxia alone, and those of the corresponding paired tissue samples undergoing a hypoxia-reoxygenation cycle, no significant differences were observed. However, a mean increase of $36 \%$ at 120 minutes was observed for both inflamed rheumatoid tissue and inflamed osteoarthritic tissue samples exposed to the hypoxiareoxygenation cycle, over and above that seen for the respective paired samples maintained under conditions of continuous hypoxia ( $\mathrm{p}=0.0086$; paired sample $t$ test).

Inclusion of the xanthine oxidase inhibitors, oxypurinol or amflutizole, with inflamed tissues subjected to hypoxia-reoxygenation resulted in $82 \%$ and $26 \%$ reduction in the signal height compared with control samples.

Table 2 shows the results of the morphometric analysis of six of the specimens and the corresponding clinical score and ESR signal height at 120 minutes after the start of the hypoxia-reoxygenation experiments. A correlation was observed between the normalised ESR signal height 120 minutes after a hypoxia-reoxygenation cycle and mean capillary density of the specimens (product 
Table 2 Normalised signal height at 120 minutes for synovial tissue samples exposed to a hypoxia-reoxygenation cycle, compared with mean capillary density and cell density

\begin{tabular}{lllll}
\hline & $\begin{array}{l}\text { Inflammation } \\
\text { score }\end{array}$ & $\begin{array}{l}\text { ESR signal height } \\
\text { (arbitrary units) }\end{array}$ & $\begin{array}{l}\text { Mean capillary } \\
\text { density }\end{array}$ & Cell density \\
\hline Inflamed RA & 4 & 1710 & $134 \cdot 3$ & +++ \\
Inflamed RA & 3 & 970 & $120 \cdot 3$ & ++ \\
Less inflamed RA & 1 & 327 & $83 \cdot 4$ & + \\
Less inflamed RA & 2 & 310 & $80 \cdot 1$ & + \\
Less inflamed OA & 1 & 249 & $76 \cdot 5$ & ++ \\
Less inflamed OA & 1 & 307 & $78 \cdot 4$ & + \\
\hline
\end{tabular}

moment correlation coefficient $r=0.97$, $\mathrm{p}<0.01$ ). No evidence for a relationship between the cell density of the specimens or the mean capillary depth and normalised ESR signal height was observed.

\section{Discussion}

Although there is ample evidence of oxidative damage in the synovial fluid components of inflamed joints, the production of oxidising species capable of generating an ESR detectable signal by synovial tissue has only been reported previously in one specimen of inflamed rheumatoid synovium. ${ }^{21}$ In that report the profile for the production of oxidising species was not studied in detail in relation to time, degree of synovial inflammation, or diagnostic category. The present study provides further evidence, using electron spin resonance spectroscopy, of the production of oxidising species from inflamed human synovium undergoing hypoxiareoxygenation cycles.

The production of oxidising species was maximal in the most inflamed specimens of synovial tissue as determined by our simple clinical scoring system. The degree of synovial inflammation appeared to be a more important determinant of oxidising capacity than the diagnostic label (either rheumatoid arthritis or osteoarthritis) attached to the patient. Only six specimens of inflamed synovia were collected in the 12 month period of study, as joint replacements are often performed at the end stage of arthritis. The small numbers thus limit the extent to which a quantitative analysis of the data may be pursued at this stage; however, it appears that an increase in the production of oxidising species after ex vivo hypoxiareoxygenation occurs in both rheumatoid and osteoarthritic inflamed synovia, but not in less inflamed synovia.

The observation that the relative concentration of reactive oxygen species detectable by ESR correlated with the mean capillary density of the synovial tissue specimens is of interest. Once more the small number of specimens restricts quantitation of the data; however, this correlation suggests that the endothelial cell is an important site for the production of reactive oxygen species by the synovium. Endothelial cells have been shown to be the main site for the localisation of xanthine oxidase/dehydrogenase ${ }^{8}$ in normal and rheumatoid synovia and this enzyme is a potent catalyst for the production of superoxide and other reactive oxygen species. Indeed, inhibition of the signal height by both oxypurinol and amflutizole in the system described underlines the contribution of endothelial cell xanthine oxidase to the enhanced production of oxidising species by inflamed synovial tissues after hypoxiareoxygenation. We suggest that the more pronounced effect of oxypurinol is caused by its relatively greater scavenging capacity at the particular dose tested in our experiments, in addition to its inhibitory effect on xanthine oxidase activity. ${ }^{22}$

We have previously proposed ${ }^{1}$ that, in the early stages of inflammation, exercise promotes cycles of hypoxia-reoxygenation which elicit the production of oxidising species that cause further damage to critical biomolecules and structures present in the joint. Resting an inflamed joint would be expected to break this cycle, which may explain the well established observation that the clinical features of an inflamed joint improve with bed rest and splinting. However, exercise-induced production of reactive oxygen species may be cytotoxic to both synoviocytes and endothelial cells of the inflamed synovium, whilst at the same time stimulating fibroblast proliferation. ${ }^{23}$ Hence, following repeated cycles of hypoxic-reperfusion injury, an 'autosynovectomy' may result and lead ultimately to the fibrotic (burnt out) phase of rheumatoid arthritis. Such a course of events may explain the paradoxical suppression of rheumatoid disease with exercise. ${ }^{24} 25$

Our results provide ex vivo evidence of increased production of reactive oxidising species with reoxygenation of inflamed hypoxic human synovial tissue compatible with the hypothesis of movement induced hypoxicreperfusion injury in the inflamed joint. In addition, these studies provide a rationale for the clinical efficacy of both rest and exercise programmes in inflammatory arthropathies. We also suggest that the application of ESR spectroscopy may provide the basis for in vitro assessment of antioxidant and antiinflammatory drugs.

We are grateful to $\mathrm{Mr}$ W J Grange, Consultant Orthopaedic Surgeon, for providing us with tissue samples from his patients Surgeon, for pr and to Dr Cliff Stevens of this department for his help with the histological studies. DS was supported by a scholarship from the Wishbone Trust of the British Orthopaedic Association. The Bone and Joint Research Unit is supported by the Arthritis and Rheumatism Council and the British Technology Group.

1 Blake D R, Allen R E, Lunec J. Free radicals in biological systems-a review orientated to inflammatory processes. systems-a review orientated to

2 Blake D R, Merry P, Unsworth J, et al. Hypoxic-reperfusion injury in the inflamed human joint. Lancet 1989; 334 injury in

3 Merry P, Grootveld M, Blake D R. Free radicals and hypoxic-reperfusion injury. A mechanism producing persistent synovitis? In: Scott $T$, Jayson $M$, Moll J, Isenberg I, eds. Topical reviews-Reports on rheumatic diseases. London: Arthritis and Rheumatism Council, 1990; 15.

4 Zhang Z, Farrell A J, Blake D R, Chidwick K, Winyard P G. Synovial fluid alpha-1-antitrypsin is inactivated by exercise in rheumatoid arthritis. FEBS Lett 1993; 321: 274-8.

5 Lunec J, Blake D R, McCleary S J, Brailsford S, Bacon P A. Self perpetuating mechanisms of immunoglobulin $G$ aggregation in theumatoid inflammation. 7 Clin Invest 1985; 76: 2084-90.

6 Grootveld M, Henderson E B, Farrell A J, Blake D R, Parkes H G, Haycock P. Oxidative damage to hyaluronate and glucose in synovial fluid during exercise of the

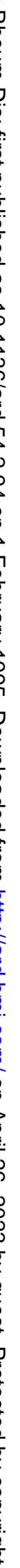


7 Rowley D A, Gutteridge J M C, Blake D R, Farr M, Halliwell B. Lipid peroxidation in rheumatoid arthritis. Thiobarbituric acid reactive material and catalytic iron salts in synovial fluid from rheumatoid patients. Clin $\mathrm{Sci}$ 1984; 66: 691-5.

8 Stevens C R, Benboubetra M, Harrison R, Sahinoglu T, Smith E C, Blake D R. Localisation of xanthine oxidase to synovial endothelium. Ann Rheum Dis 1991; 50 $760-2$

9 Allen R E, Outhwaite J, Morris C J, Blake D R. Xanthine oxido-reductase is present in human synovium. $A n n$ Rheum Dis 1987; 46: 843-5.

10 McCord J M. Oxygen-derived free radicals in postischaemic tissue injury. N Engl f Med 1985; 312: 159-63.

11 Symons M. Chemical and biochemical aspects of electron spin resonance spectroscopy. Wokingham: Van Nostrand resonance spectroscopy.

12 Finkelstein E, Rosen G M, Rauckman E J. Spin trapping of superoxide and hydroxyl radical: practical aspects. Arch of superoxide and hydroxyl radical: practical aspects. Arch

13 Samuni $A$, Samuni nitrobenzene sulfonate as a spin trap in biological systems Free Radic Biol Med 1989; 7: 37-43.

14 Nazhat N B, Yang G, Allen R E, Blake D R, Jones P. Does 3,5 dibromo-nitrosobenzene sulphonate spin trap superoxide radicals? Biochem Biophys Res Commun 1990; 166: $807-12$.

15 Wolfenden B S, Willson R L. Radical cations as reference chromogens in kinetic studies of one electron transfer reactions: pulse radiolysis studies of ABTS. $\mathcal{f}$ Chem Soc Perkin Trans II 1982; 805-11.

16 Flitter W D, Mason R P. The horseradish peroxidase catalysed oxidation of deoxyribose sugars. Free Radic Res Commun 1990; 9: 297-302.
17 Roselaar S E, Nazhat N B, Winyard P G, Blake D R, Cunningham $\mathrm{J}$. An oxidizing middle molecule accumulates in acute and chronic renal failure: detection by ESR spectroscopy. F Am Soc Nephrology 1992; 3: 348.

18 Hoey B M Butler J, Halliwell B On the specificity of allopurinol and oxypurinol as inhibitors of xanthine oxidase: a pulse radiolysis determination of the rate constants dase: a pulse radiolysis determination of the rate constants
for the reaction of allopurinol and oxypurionol with hyfor the reaction of allopurinol and oxypurionol with hy-

19 Werns S W, Grum C M, Ventura A, et al. Xanthine oxidas does not limit canine infarct size. Circulation 1991; 83: 995-1005.

20 Stevens C R, Blake D R, Merry P, Revell P A, Levick J R. A comparative study by morphometry of the microvasculature in normal and rheumatoid synovium. Arthritis Rheum 1991; 34: 1508-13.

21 Allen R E, Blake D R, Nazhat N B, Jones P. Superoxide radical generation by inflamed human synovium after radical generation by inflamed

22 Moorhouse P C, Grootveld M, Halliwell B, Quinlan J G. Allopurinol and oxypurinol are hydroxyl radical cavengers.

23 Murrell G A C, Francis M J O. Free radicals, fibroblasts and proliferation. In: Rice-Evans C, ed. Free radicals, disease states and anti-radical interventions. London: Richelieu Press, 1989; 247-63.

24 Harkcom T M, Lampman R M, Banwell B F, Castor C W. Therapeutic value of graded aerobic exercise training in rheumatoid arthritis. Arthritis Rheum 1985; 28: 32-9.

25 Lyndberg K, Danneskiold-Samsoe B, Halskov O. The effect of physical training on patients with rheumatoid arthritis: changes in disease activity, muscle strength and aerobic capacity. A clinically controlled minimized cross-over study. Clin Exp Rheumatol 1988; 6: 253-60. 